\title{
Detection of Demagnetization in Permanent Magnet Synchronous Machines Using Hall-Effect Sensors
}

\author{
David Reigosa ${ }^{*}$, Daniel Fernández ${ }^{*}$, Yonghyun Park ${ }^{* *}$, Alberto B. Diez ${ }^{*}$, Sang Bin Lee ${ }^{* *}$ and Fernando Briz ${ }^{*}$ \\ "University of Oviedo. Dept. of Elect., Computer \& System Engineering, Gijón, 33204, Spain. \\ Korea University, Dept. of Elect. Engineering, Seoul, Korea. \\ diazdavid@uniovi.es, fernandezalodaniel@uniovi.es, yonghyun.park@eecs.korea.ac.kr, alberto@isa.uniovi.es, \\ sangbinlee@korea.ac.kr, fernando@isa.uniovi.es
}

\begin{abstract}
Torque production capability of permanent magnet synchronous machines (PMSMs) depends on the magnetization state of the permanent magnets (PMs). Electrical stress, thermal stress, or the combined effect of both can produce demagnetization of the PMs, which eventually can result in several adverse effects including decrease in the motor torque and efficiency, and increase of the torque ripple and vibration, eventually degrading the performance and reliability of the motor and drive system. A number of approaches have been proposed for detecting PM demagnetization using model-based flux estimation, signal injection, spectrum analysis of current/back-EMF; however, all these methods show limitations in terms of invasiveness, implementation cost and/or reliability of the diagnosis. In this paper, accurate PM demagnetization detection based on measurements from hall-effect sensors is proposed. Such sensors are often mounted in commercial PMSMs, the proposed method can therefore be implemented at practically no cost. $^{1}$
\end{abstract}

Index Terms - Permanent magnet synchronous machines, demagnetization, magnetization state estimation, Hall-effect sensors.

\section{Introduction}

Permanent magnet synchronous machines (PMSMs) have been the focus of significant research efforts due to their high efficiency, high power density, good dynamic response, and ease of control compared with other types of machines [1][22]. Since the torque production capability of PMSMs directly depends on the magnetization state of PMs [1]-[2], methods for detecting PM demagnetization at an early stage have been intensively investigated [3]-[22]. Demagnetization is typically caused by a combination of electrical and thermal stresses [3]-[4]. Demagnetization in PMs can occur locally causing partial demagnetization, or globally resulting in uniform demagnetization [3]. Local demagnetization can potentially reduce the motor torque and efficiency, also increasing the harmonic content of the stator current, producing torque ripple and vibration. Global demagnetization has a more severe impact on the average torque and efficiency. Therefore, early detection of PM demagnetization detection is of great importance.

\footnotetext{
${ }^{1}$ This work was supported in part by Spanish Ministry of Education, Culture and Sports through "José Castillejo Program" under grant PX15/00354, by Regional Ministry of Education, Culture and Sport of the Principality of Asturias through "Severo Ochoa Program" under Grant BP-13067 and by the Research, Technological Development and Innovation Programs of the Spanish Ministry Economy and Competitiveness, under grant MINECO-17ENE2016-80047-R.
}

Magnetization state of the PMs can be measured or estimated. Magnetization state can be directly measured on the surface of the rotor using a gauss meter [4]-[5], however, this normally requires motor disassembly or even rotor removal. A PM field distribution measurement system for IPMSMs was presented in [6], which provided precise and high bandwidth measurements of the PM field without interfering with the normal operation of the machine. Unfortunately, the use of such system is not viable in commercial applications due to both cost and installation issues. In conclusion, systems for direct PM field measurement suitable for their use in industry are not available to the best of authors' knowledge.

Alternatively to direct measurement, magnetization state can be estimated to detect either global or partial demagnetization. Global demagnetization detection methods that have been proposed include the use of backelectromotive-force (BEMF) [7]-[9], pulse injection [10], and high-frequency signal injection [11]-[12]. BEMF and pulse injection methods require the machine to be rotating, estimation with the motor at standstill not being possible. High-frequency signal injection methods can operate in the whole speed range. These methods estimate the PM magnetization state from the PM high-frequency resistance. However, these methods place concerns due to the potential adverse effects of the injected HF signal. Previous methods [7]-[12] provide an average estimate of PM magnetization state and therefore are not able to distinguish between global and local demagnetization. A wide variety of local demagnetization detection methods have been proposed [13][23], including stator current analysis [13]-[17], zero-sequence voltage [18], BEMF [19], vibration analysis [20]-[21], change in the shaft trajectory [22], or signal injection [23] methods. Stator current analysis, zero-sequence voltage, BEMF, vibration and shaft trajectory methods require the machine to be rotating, whereas signal injection methods require the machine to be at standstill. Among online methods, stator current analysis [13]-[17] and BEMF [19] are the most appealing option, as they use the stator terminal variables, and do not require installation of additional sensors. A limitation of online spectrum analysis based methods [13]-[23] is that mechanical issues, such as eccentricity, load oscillations/variations or misalignments can induce frequency components in the stator current identical to that of local demagnetization, which makes it very difficult to distinguish between mechanical issues and local demagnetization.

Commercial PMSMs are often equipped with hall-effect field sensors intended for initial position estimation, their use 


\begin{tabular}{|l|l|}
\hline \multicolumn{2}{|c|}{ Table I. Machine parameters } \\
\hline$P_{\text {RATED }}(\mathrm{kW})$ & 7.5 \\
\hline$I_{\text {RATED }}(\mathrm{A})$ & 14 \\
\hline$\omega_{\text {RATED }}(\mathrm{rpm})$ & 1800 \\
\hline Stator slots & 36 \\
\hline Poles & 6 \\
\hline Rotor radius (mm) & 54.2 \\
\hline Magnets & $\mathrm{N} 42 \mathrm{SH}$ \\
\hline Magnet dimensions: width, height and length (mm) & $42 \times 6 \times 10$ \\
\hline Magnet position from shaft center (mm) & 44.6 \\
\hline Magnet position, $x y z(\mathrm{~mm})$ & $0,0,6$ \\
\hline Airgap length (mm) & 0.8 \\
\hline Inner stator radius $(\mathrm{mm})$ & 55 \\
\hline Outer stator radius $(\mathrm{mm})$ & 88 \\
\hline
\end{tabular}

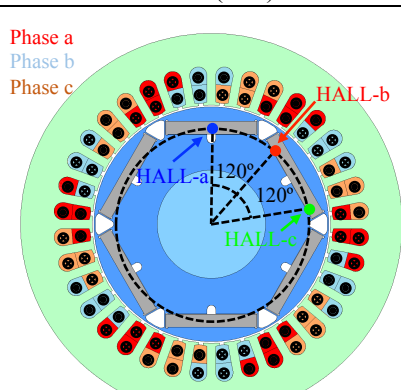

a)

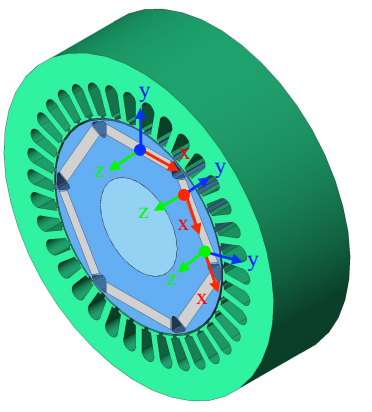

b)
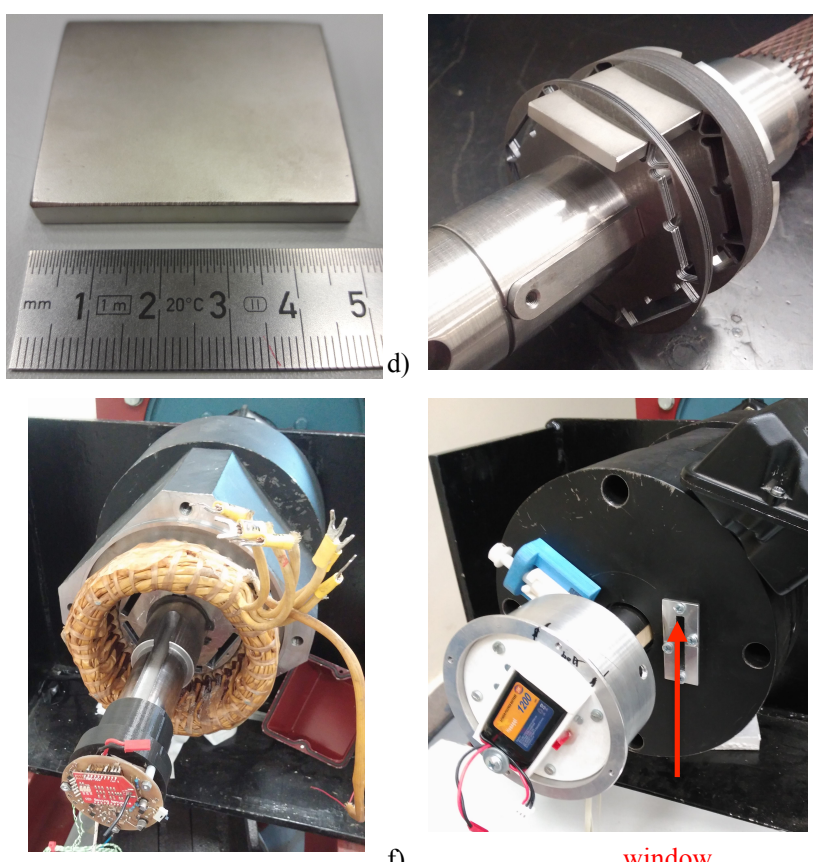

e)

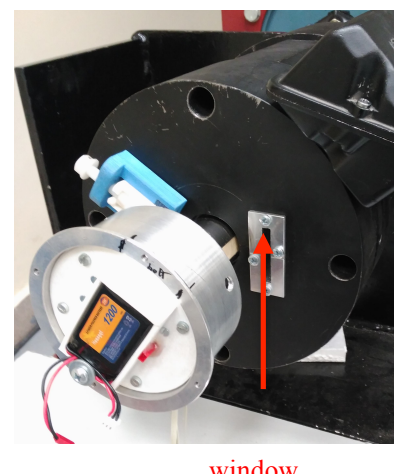

f)

window

Fig. 1.- Schematic representation of the machine. a) sensors location, b) coordinate system for each sensor ( $x=$ tangential direction, $y=$ radial direction, $z=$ axial direction), c) Nedimioum Neomax 42SH PM $(42 * 6 * 50 \mathrm{~mm})$, d) rotor lamination assembling, e) machine assembling and $\mathrm{f}$ ) end shield modification.

for torque and motion control has also been reported [24]-[32]. The hall-effect sensors can be digital [24]-[27] or analog [28][32]. Detection of PM demagnetization using hall-effect sensors is investigated in this paper [35]. The method detects demagnetization by direct measurement of the PM flux, instead of by means of indirect measurements (stator terminal voltages/currents, vibration, shaft trajectory...) used in the methods reported in the literature [7]-[23]. This helps improve sensitivity and reliability since interference due to non-fault related factors and sensitivity to model or parameters can be minimized. The proposed method is intended to use halleffect sensors already available in many commercial PMSMs and does not affect therefore the machine design, performance, or cost.

The paper is organized as follows: analysis of the measurements provided by digital and analog hall-effect sensors used in PMSMs is presented in section II, principles of demagnetization detection using both types of hall-effect sensors and experimental results to demonstrate the viability of the concepts are presented in section III. A discussion of practical implementation issues is presented in section IV, while conclusions are provided in section V.

\section{Flux measurement in PMSMs using hall-effect sensors}

This section studies the behavior of digital and analog halleffect sensors mounted in PMSMs. The schematic design of the machine that will be used for the study is shown in Fig. 1a and $1 \mathrm{~b}$. Dimensions and ratings of the test machine are shown in Table I. Fig. 1c shows a Neomax 42SH PMs with rated magnetic flux density of $1.33 \mathrm{~T}$, which was used in the test machine. Fig. 1d shows the rotor lamination assembling, Fig. 1e shows the machine final assembling and Fig. 1f shows the end shield modified (see rectangular window) to allow insertion/extraction of the magnets without the need to remove the rotor. This is key to make viable the evaluation of magnets with a large variety of magnetization states.
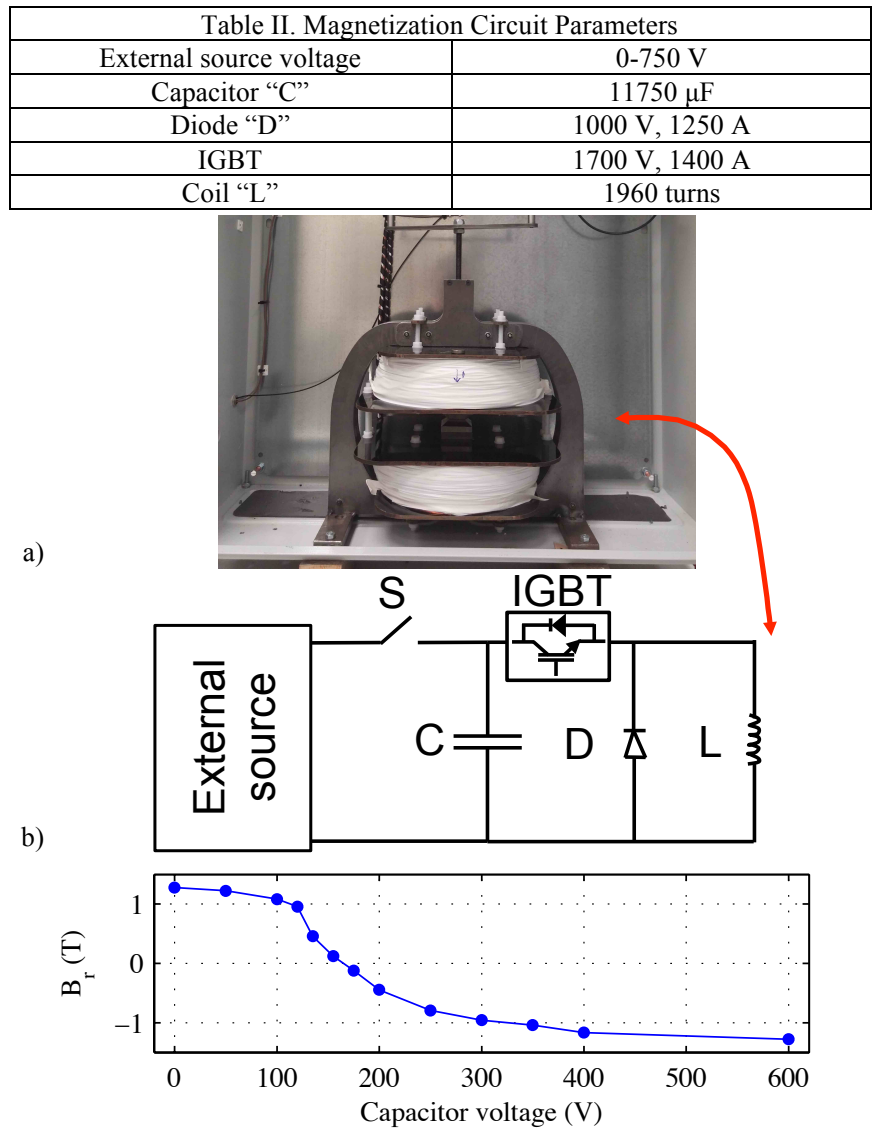

Fig. 2.- a) Pulse magnetizer, b) schematic representation of the circuit used for PM magnetization and demagnetization, c) and PM remnant flux $\left(\mathrm{B}_{\mathrm{r}}\right)$ depending on the capacitor voltage. 


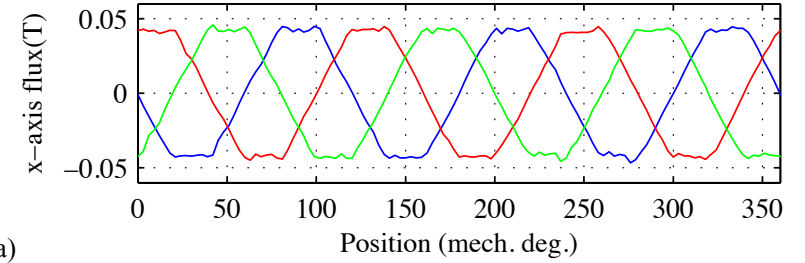

a)

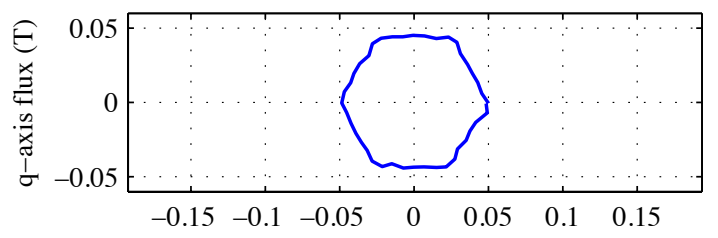

b)

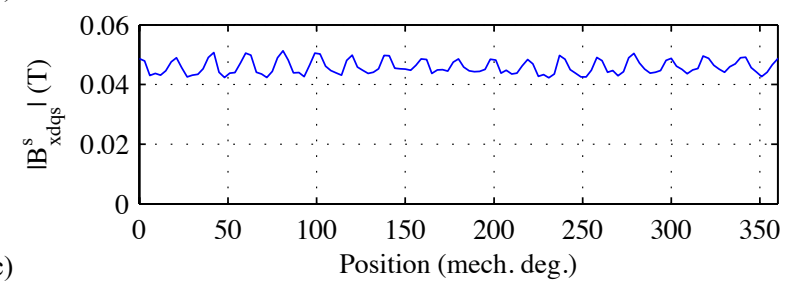

c)

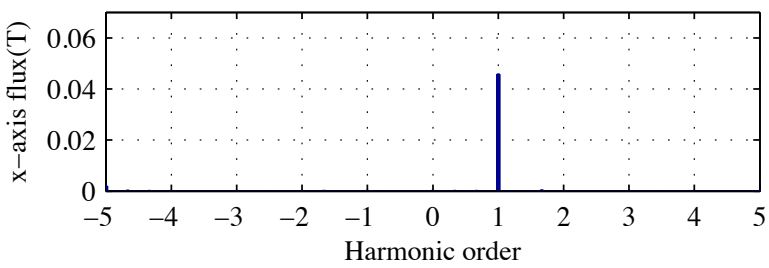

Fig. 3.- a) Measurements in the $x$-axis direction by all the three analog halleffect sensors ( $B_{x a}, B_{x b}$ and $B_{x c}$ ), b) resulting magnetic flux density complex vector, $\bar{B}_{x d q s}^{s}$, c) magnitude of $\bar{B}_{x d q s}^{s}$, d) and FFT of $\bar{B}_{x d q s}^{s}$. Note the logarithmic scale for the FFT magnitude. $x=0 \mathrm{~mm}, y=0 \mathrm{~mm}, z=5 \mathrm{~mm}$, $\omega_{r}=1 \mathrm{pu}$ and $i_{d q}=0 \mathrm{pu}$, case \#1 in Table III, with all PMs fully magnetized.

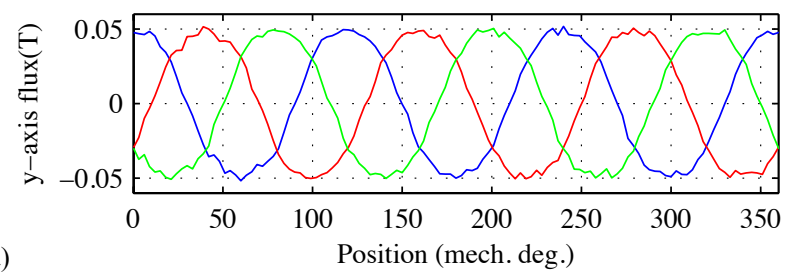

a)

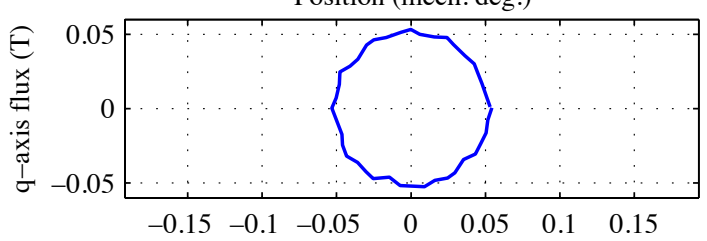

b)

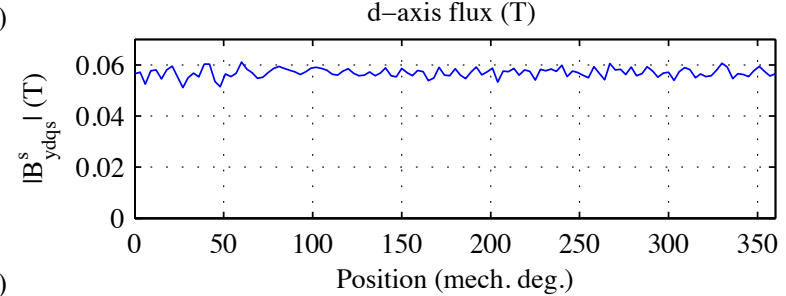

c)

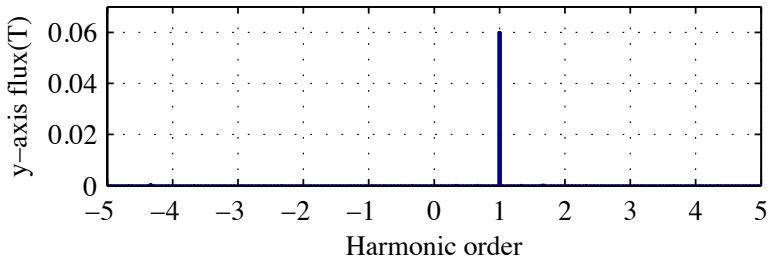

d) Harmonic order

Fig. 4.- Same results as in Fig. 3 but for the $y$-axis direction.
Hall-effect sensors normally used in PMSM drives measure the magnetic flux density along a single direction (i.e. 1D sensors), $y$ direction in the coordinate system shown in Fig. $1 \mathrm{~b}$ being normally used [24]-[25]. For analysis purposes, field sensors that measure the magnetic flux density in $x, y$ and $z$ directions (3D sensors) will be used in this paper. The purpose of this is to study the sensitivity of the proposed method to the orientation of the sensor. It has been concluded from the preliminary analysis, that the magnetic flux density in the $z$-axis direction is not useful for detecting PM demagnetization, the measurements in this direction not being shown therefore in this paper.

For the experiments shown in this paper, PMs are first magnetized to the desired level using the pulse magnetizer shown in Fig. 2 and then inserted in the machine. The parameters of the magnetization circuit are shown in Table II. The magnetization state of the PMs can be changed between 1 to $1 \mathrm{pu}$; uniform and non-uniform magnetization being feasible.

\section{II.A Healthy machine (PMs fully magnetized)}

The measured magnetic flux density by the three analog hall-effect sensors (see Fig. 1a and 1b) in the $x$-axis direction for one mechanical rotor revolution (which corresponds to three electrical periods) and without injecting stator current (i.e. $i_{d q}=0 \mathrm{pu}$ ) are shown in Fig. 3a. The use of complex vector notation for the analysis of the field measured by all the three hall-effect sensors can provide relevant advantages, compared to the option of analyzing the signals individually. The magnetic flux density complex vector $\bar{B}_{m d q s}^{s}$, is defined as (1), where $m$ stands for either $x$ or $y$-axis and $B_{m a}, B_{m b}$ and $B_{m c}$, are the magnetic flux densities measured by sensors $a, b$ and $c$ along the $m$ direction respectively.

$\bar{B}_{m d q s}^{s}=2 / 3\left(B_{m a}+a B_{m b}+a^{2} B_{m c}\right)$

The trajectory of the resulting magnetic flux density complex vector in the $x$-axis direction is shown in Fig. $3 \mathrm{~b}$. A nearly hexagonal trajectory is observed.

The magnitude of $\bar{B}_{x d q s}^{s}$ and the corresponding FFT are shown in Fig. 3b-c, respectively. It can be observed from Fig. 3c that $\left|\bar{B}_{x d q s}^{s}\right|$ has a mean magnitude of $\approx 0.046 \mathrm{~T}$ with a peak-topeak value of $\approx 0.01 \mathrm{~T}$ (see Table III, case \#1). The spectrum in Fig. $3 \mathrm{~d}$ consists of a fundamental harmonic component, which rotates at the machine speed, and additional harmonic components. The fundamental component is in principle the desired component, as it is used for continuous torque production, while the harmonics are in principle undesired components, as they will produce torque pulsations, additional losses, etc. $\bar{B}_{m d q s}^{s}$ can be expressed as (2), where $\omega_{r}$ is the machine speed, $B_{m d g s_{-} 1 \omega r}^{s}$ is the magnitude of the fundamental harmonic component, $B_{m d q s_{-} n \omega r}^{s}$ is the magnitude of the " $n$ th" harmonic component, $\varphi_{m}$ is the angle between the field produced by the stator current (which has a radial direction) and the measuring direction of field sensors, i.e $\varphi_{x}=90$ ( $x$-axis) and $\varphi_{y}=0$ (y-axis), and $\varphi_{n m}$ is the angular offset of the $n^{\text {th }}$ harmonic component.

$\bar{B}_{m d q s}^{s}=B_{m d q s_{-} 1 \omega r}^{s} e^{j\left(\omega_{r} t+\varphi_{m}\right)}+\sum_{n} B_{m d q s_{-} n \omega r}^{s} e^{j\left(n \omega_{r} t+\varphi_{m m}\right)}$ 


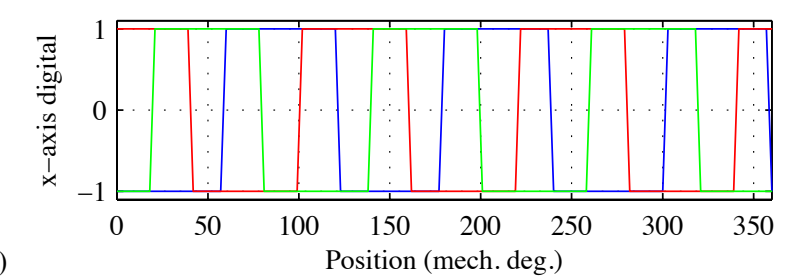

a)

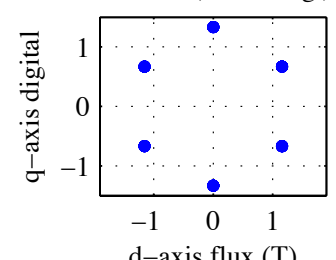

b)

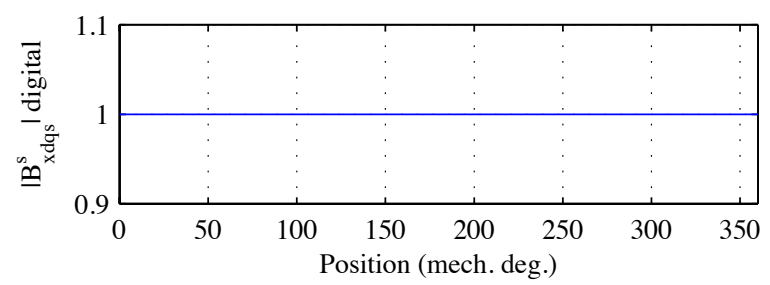

c)

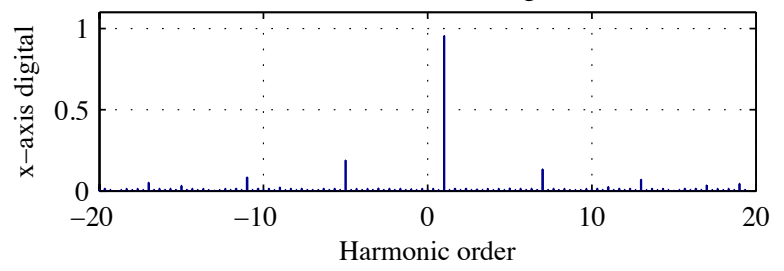

Fig. 5.- a) Measurements in the $x$-axis direction by all the three digital halleffect sensors ( $B_{x a}, B_{x b}$ and $B_{x c}$ ), b) resulting magnetic flux density complex vector, $\bar{B}_{x d q s}^{s}$, c) magnitude of $\bar{B}_{x d q s}^{s}$, d) and FFT of $\bar{B}_{x d q s}^{s}$. Note the logarithmic scale for the FFT magnitude. $x=0 \mathrm{~mm}, y=0 \mathrm{~mm}, z=5 \mathrm{~mm}$, $\omega_{r}=1 \mathrm{pu}$ and $i_{d q}=0 \mathrm{pu}$, case \#1 in Table III, with all PMs fully magnetized.

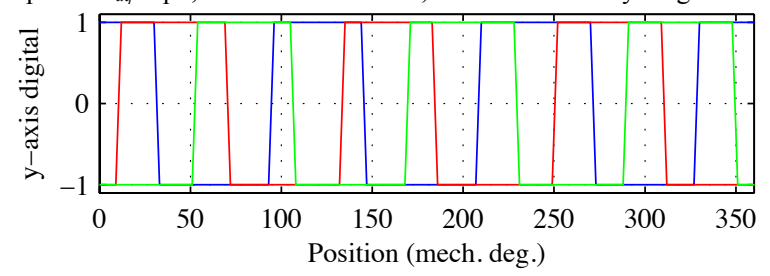

a)

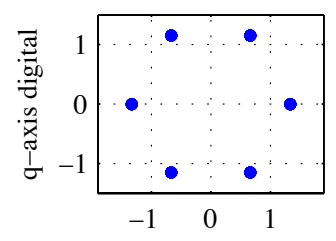

b)

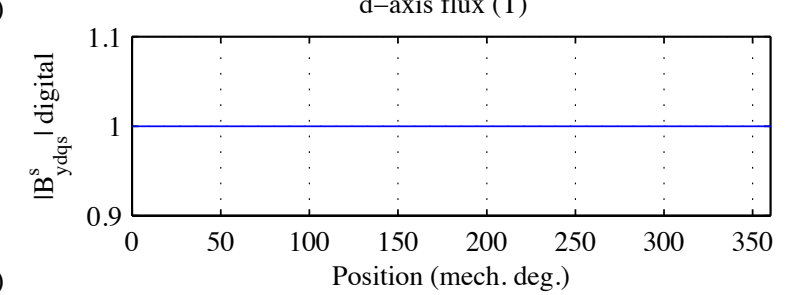

c)

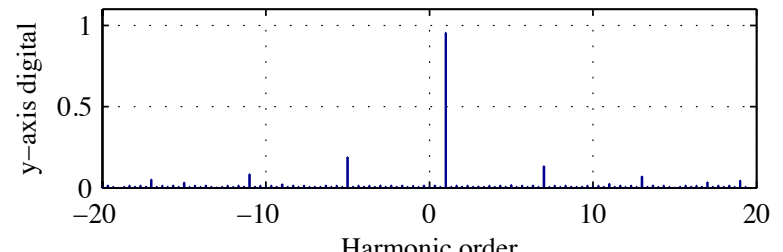

d)

Harmonic order

Fig. 6.- Same results as in Fig. 5 but measuring the field in the $y$-axis direction.

From (2), the total harmonic distortion (THD) can be used to assess the signal (fundamental component) to noise (additional harmonics) ratio. The THD for the case shown in Fig. 3 is $5.63 \%$. Fig. 4 shows the same results as in Fig. 3 but for the $y$-axis direction (see Fig. 1). The mean value of $\left|\bar{B}_{y d q s}^{s}\right|$ is $\approx 0.054 \mathrm{~T}$ in this case, with a peak-to-peak value of $\approx 0.0065 \mathrm{~T}$ and a THD of $3.98 \%$.

The measurements and processing shown in Fig. 3 and 4 where repeated using digital hall-effect sensors, the corresponding results being shown in Fig. 5 and 6 . The resulting magnetic flux density complex vector both for the $x$ and $y$-axis is now a hexagon, as observed in Fig. $5 \mathrm{~b}$ and $6 \mathrm{~b}$. The FFT of $\bar{B}_{x d q s}^{s}$ (see Fig. 5d) is seen now to consists of a fundamental harmonic component which rotates at the machine speed and harmonics components of orders $n=-5,7$,$11,13 \ldots$ whose magnitude is inversely proportional to the harmonic order. The THD of $\bar{B}_{x d q s}^{s}$ is $31.16 \%$ in this case. Fig. 6 shows the same results as Fig. 5 but for the $y$-axis direction. Case \#1 in Table III summarizes all the results using both analog and digital field sensors.

\section{Demagnetization detection using hall-effect sensors}

Analysis of the signals provided by the hall-effect sensors for the case of healthy machines has been presented in the previous section. The use of these signals for demagnetization detection is discussed in this section. A simple model able to predict the effect of demagnetization on the measurements provided by the field sensors is developed in subsection III- $A$. Experimental verification is presented in further subsections.

\section{III.A Flux profile of partially demagnetized PM modeling}

Fig. 7a shows the flux measured by the three analog halleffect sensors ( $B_{y a}, B_{y b}$ and $B_{y c}$ ) in the $y$-axis direction for the case of an ideal machine. The signals consist of a purely sinusoidal component. This is seen to be in good agreement with the results shown in Fig. 4. Fig. 7b shows the FFT of $\bar{B}_{y d q s}^{s}$ (1), which is seen to consist of a single harmonic component of the type $B_{y d q s_{-} 1 \omega r}^{s} e^{j\left(\omega_{r} t+\varphi_{m}\right)}$, i.e. which rotates at the machine speed.

In the event case of a machine with some demagnetized PM, the flux measured by the analog hall-effect sensors in the $y$-axis direction should be reduced when the demagnetized PM faces each sensor compared to the healthy PM. This behavior can be modeled by multiplying signals shown in Fig. $7 \mathrm{a}$, by a square-type window function. The value of this window function is 1 for the angles in which healthy PM face the sensor, and will have a value $<1 \mathrm{pu}$ when demagnetized PMs face the sensor. Window functions for sensors $a, b$ and $c$ ( $W_{y a}, W_{y b}$ and $W_{y c}$ ) will be shifted by 120 electrical degrees, as this is the angle among sensors. An example of such window functions is shown in Fig. 7c. Fig. 7d shows the FFT of the window function, which will be used later. For a 6-pole machine and for the case of a single partially demagnetized $\mathrm{PM}$, the window function is $<1$ only for one semicycle of the sinusoidal wave (see Fig. 7e). Fig. 7e shows the magnetic flux densities that result from multiplying the flux measurement for the case of a healthy machine shown in Fig. 7a by the window functions in Fig. 7c, (3)-(5). Finally, Fig. $7 \mathrm{f}$ shows the FFT of $\bar{B}_{y d q s}^{s}$ (resulting form $B_{y a}, B_{y b}$ and $B_{y c}$ in Fig. 7e, i.e. 


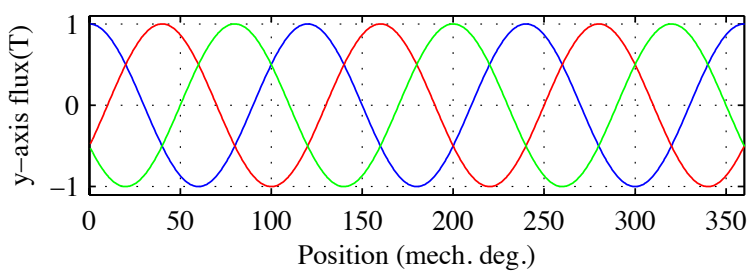

a)

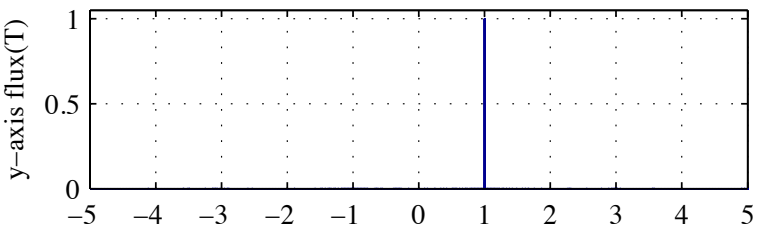

b)

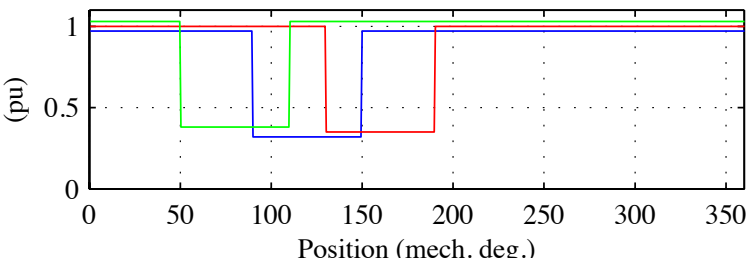

c)

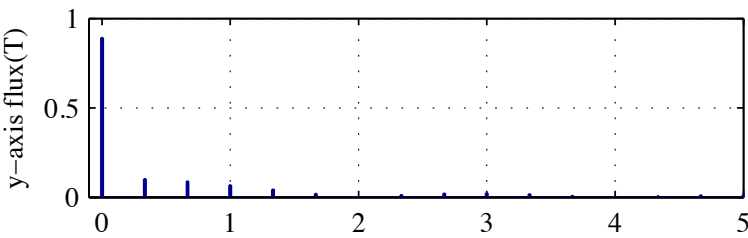

d)

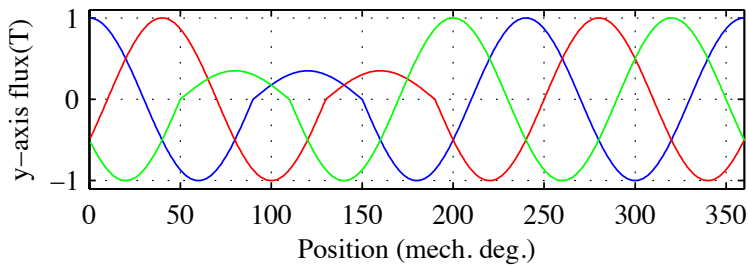

e)

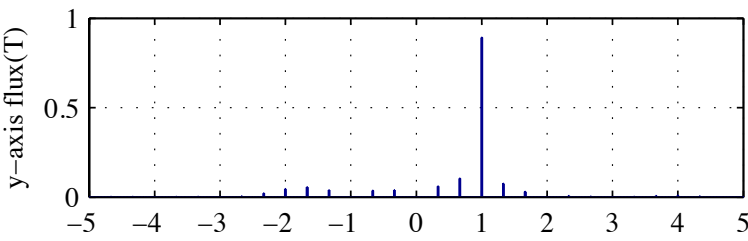

f)

Harmonic order

Fig. 7.- a) Ideal flux measurements in the $y$-axis direction by all the three analog hall-effect sensors ( $B_{y a}, B_{y b}$ and $B_{y c}$ ) in a healthy machine, b) FFT of $\bar{B}_{y d q s}^{s}$ for a healthy machine (a)), c) ideal flux variation due to demagnetization, d) FFT of the waveform shown in c), e) example of ideal measurements in the $y$-axis direction by all the three analog hall-effect sensors for the case when 1 PM is demagnetized and f) FFT of $\bar{B}_{y d q s}^{s}$ for a machine with 1 PM demagnetized (e)). $\omega_{r}=1 \mathrm{pu}, 1 \mathrm{PM}$ demagnetized (case \#7 in Table III).

faulty machine). It is observed that the spectrum of $\bar{B}_{y d q s}^{s}$ for the case of a machine with a demagnetized PM includes additional harmonics compare to the case of a healthy machine. The harmonic content in Fig. $7 \mathrm{f}$ was expected, as the multiplication in the time domain, in (6) corresponds to the convolution in the frequency domain describe by (7), where * is the convolution operator.

$$
\begin{aligned}
& B_{y a}^{\prime}=B_{y a} W_{y a} \\
& B_{y b}^{\prime}=B_{y b} W_{y b}
\end{aligned}
$$

$$
\begin{aligned}
& B_{y c}^{\prime}=B_{y c} W_{y c} \\
& \begin{aligned}
\bar{B}_{m d q s}^{s}= & 2 / 3\left(B_{y a} W_{y a}+a B_{y a} W_{y a}+a^{2} B_{y a} W_{y a}\right) \\
= & 2 / 3\left(B_{y a}^{\prime}+a B_{y b}^{\prime}+a^{2} B_{y c}^{\prime}\right)
\end{aligned} \\
& F F T\left(\bar{B}_{m d q s}^{s}\right)=2 / 3\left(\begin{array}{l}
F F T\left(B_{y a}\right) * F F T\left(W_{y a}\right) \\
+a\left(F F T\left(B_{y a}\right) * F F T\left(W_{y a}\right)\right) \\
+a^{2}\left(F F T\left(B_{y a}\right) * F F T\left(W_{y a}\right)\right)
\end{array}\right)
\end{aligned}
$$

It is concluded from the previous discussion that partial demagnetization of one PM is expected to produce in the $y$ axis the wave shapes shown in Fig. 7e, the spectrum of the resulting flux vector being shown in Fig. $7 \mathrm{f}$.

Unfortunately, the effect of a demagnetized PM in the $x$ axis measurements cannot be modeled easily as it is influenced by rotor design aspects such as $q$-axis flux bridges; analysis in this case will be based on experimental measurements.

\section{III.B Experimental verification of a partially demagnetized $\underline{P M}$}

Experimental results showing the effects of partial demagnetization of a PM on the measured flux are presented in this section. Fig. 8 shows the same results as Fig. 4 when one of the magnets (PM \#5 in Fig. 1), is partially demagnetized. This corresponds to case \#7 in Table III. It can be observed the measurements shown in Fig. $8 \mathrm{a}$ are in good agreement with the predictions shown in Fig, 7e. Also, the frequency spectrum of $\bar{B}_{y d q s}^{s}$ shown in Fig. 7f, is in good agreement with the experimental results shown in Fig. $8 \mathrm{~d}$.

While the effects of demagnetization on the measurements in the $y$-axis has been shown to be relatively easy to model, analysis of the effects on the measurements in the $x$-axis direction is not straightforward. Therefore, only experimental results are shown in this case.

Fig. 8 and Fig. 9 show the same results as Fig. 3 and Fig. 4 when one of the magnets ( $\mathrm{PM} \mathrm{\# 5} \mathrm{in} \mathrm{Fig.} \mathrm{1),} \mathrm{is} \mathrm{partially}$ demagnetized. It can be seen from Fig. 9a that peak values and zero crossing of the waveforms remain almost invariant for the $x$-axis, though changes in the magnetic flux density waveforms are readily visible. On the other hand, effects on the measurements in the $y$-axis direction (Fig. 8a) are significantly more noticeable. It is deduced from Fig. 8 and Fig. 9 that measurements in the $y$-axis are more sensitive to demagnetization compared to the $x$-axis, being therefore the preferred direction.

It can be observed from Fig. $8 \mathrm{~b}$ and Fig. $9 \mathrm{~b}$ that $x$ and $y$-axis magnetic flux density complex vectors trajectory, $\bar{B}_{x d q s}^{s}$ and $\bar{B}_{\text {ydqs }}^{s}$, do not exhibit a symmetric pattern anymore. The frequency spectrums shown in Fig. $3 \mathrm{c}$ and Fig. $4 \mathrm{c}$ reveal a reduction of the mean magnitude of $\left|\bar{B}_{\text {mdqs }}^{s}\right|$ together with an increase of $\left|\bar{B}_{m d q s}^{s}\right|$ peak-to-peak value compared to the healthy machine case. It can be also observed from Fig. $8 \mathrm{~d}$ and Fig. $9 \mathrm{~d}$ that the harmonic content of $\bar{B}_{y d q s}^{s}$ and $\bar{B}_{x d q s}^{s}$ significantly increase compared to that of the healthy machine case. The asymmetric fault results in an increase of the THD of $\bar{B}_{x d q s}^{s}$ from $5.63 \%$ to $29.22 \%$, and from $3.98 \%$ to $34.70 \%$ for $\bar{B}_{y d q s}^{s}$. 


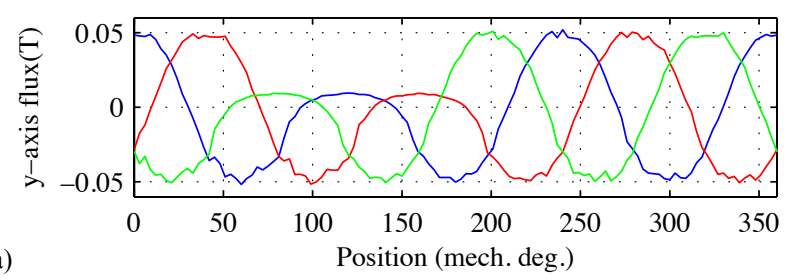

a)

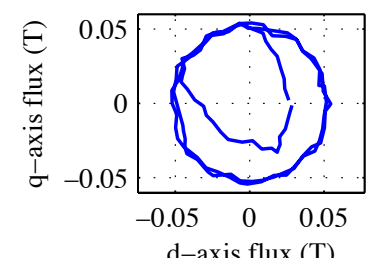

b)
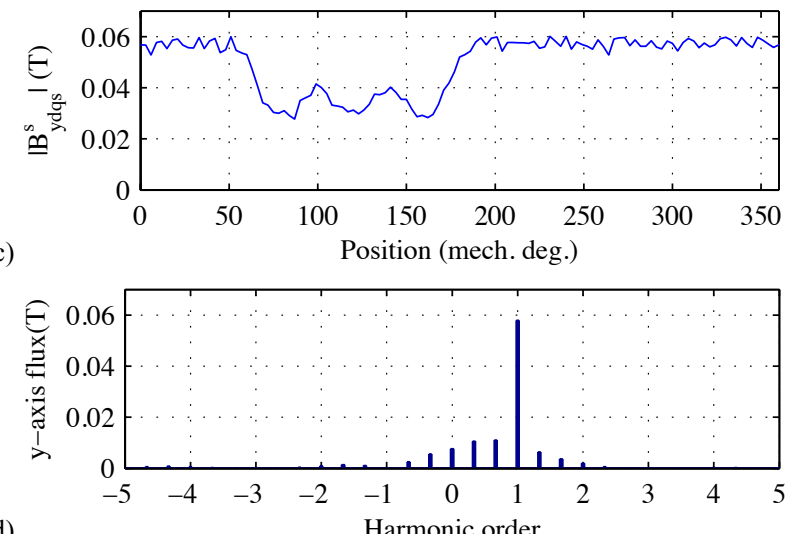

d)

$$
\text { Harmonic order }
$$

Fig. 8.-. Same results as in Fig. 3 for the case when PM \#5 magnetization (see Fig. 1) is $0.5 \mathrm{pu}(0.64 \mathrm{~T}) . \mathrm{x}=0 \mathrm{~mm}, \mathrm{y}=0 \mathrm{~mm}, \mathrm{z}=5 \mathrm{~mm} . \omega_{r}=1 \mathrm{pu}$ and $i_{d q}=0 \mathrm{pu}$, case \#7 in Table III.

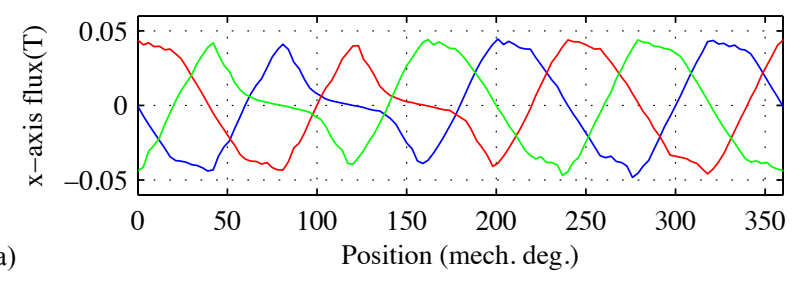

a)

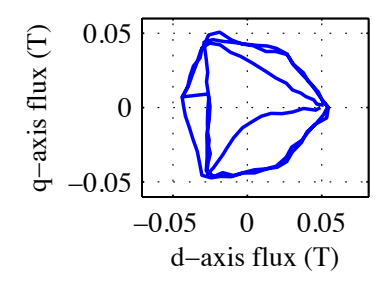

b)
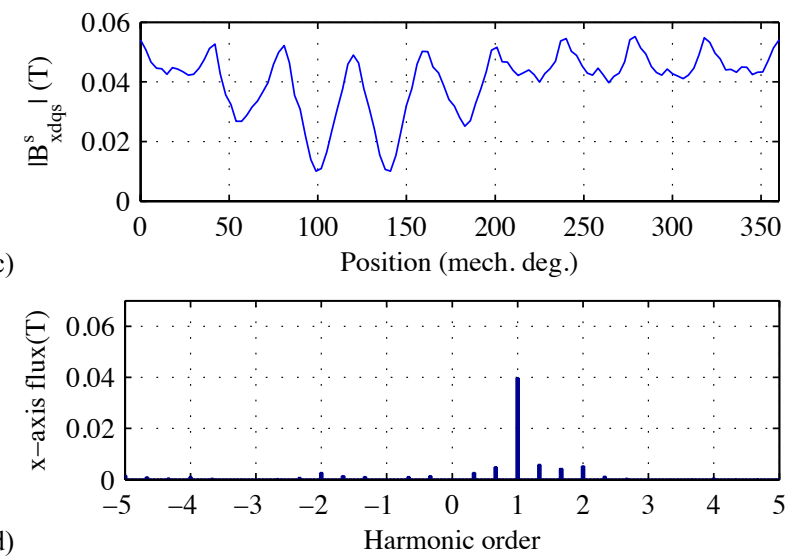

Fig. 9.- Same results as in Fig. 8, measuring the field in the $x$-axis direction.
It is concluded that either peak-to-peak or frequency content based analysis, e.g. THD, using the signals provided by analog hall effect sensors, allow reliable demagnetization detection, independent of whether the sensors are aligned with the $x$ or $y$-axis direction.

Fig. 10 and Fig. 11 show the same results as Fig. 5 and Fig. 6 when PM \#5 (see Fig. 1) is partially demagnetized and digital hall-effect sensors are used. The differences between the healthy machine (Fig. 5a) and the machine with a demagnetized PM (Fig. 10a) cases are almost negligible. This is due to the digitalization process, as demagnetization barely affects to the zero crossing of the signal. Consequently, differences in the harmonic content for the digital signal $\bar{B}_{x d q s}^{s}$ between healthy (Fig. 5d) and faulty (Fig. 10d) machine, as well as differences in the THD, are almost negligible as well. It can be concluded that digital measurements along $x$-axis direction are inadequate to detect demagnetization. Measurements in the $y$-axis direction using digital sensors show slight differences between the healthy (Fig. 6a) and demagnetized (Fig. 11a) cases; the increase of the harmonic content of $\bar{B}_{y d q s}^{s}$ for the faulty machine resulting in a modest increase of the THD (from 31.16 to $33.11 \%$ ).

Fig. 12a shows the THD of $\bar{B}_{\text {mdqs }}^{s}$ using digital hall-effect sensors when the magnetization state of PM \#5 changes from $100 \%$ to $50 \%$ (cases \#1-\#7 in Table III); differences in the THD being negligible. This confirms the inadequacy of digital hall-effect sensors aligned with the $\mathrm{x}$-axis to detect demagnetization, the sensitivity when they are aligned with the $\mathrm{y}$-axis being also modest.

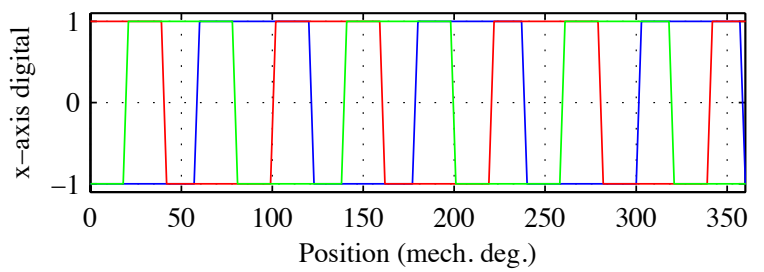

a)

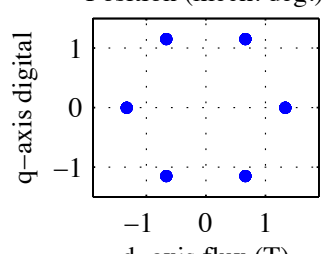

b)
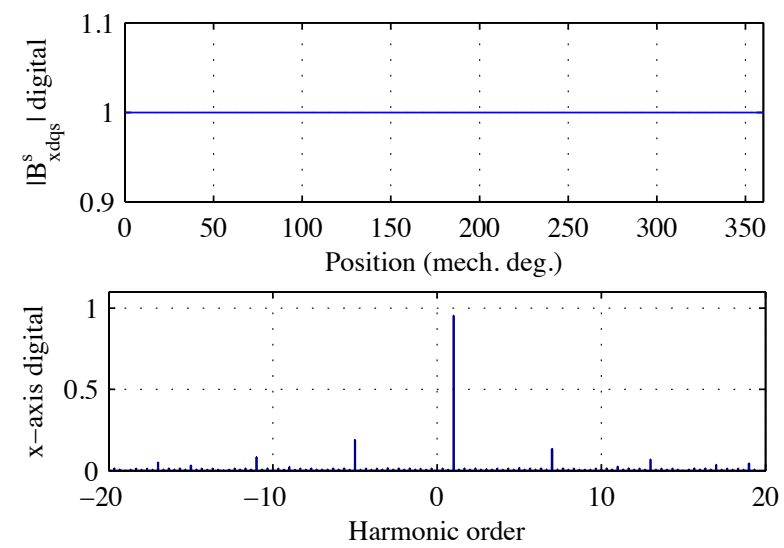

d)

Harmonic order

Fig. 10.- Same results as in Fig. 5 for the case when PM \#5 (see Fig. 1) magnetization is $0.5 \mathrm{pu}(0.64 \mathrm{~T}) . \mathrm{x}=0 \mathrm{~mm}, \mathrm{y}=0 \mathrm{~mm}, \mathrm{z}=5 \mathrm{~mm} . \omega_{r}=1 \mathrm{pu}$ and $i_{d q}=0 \mathrm{pu}$, case \#7 in Table III. 


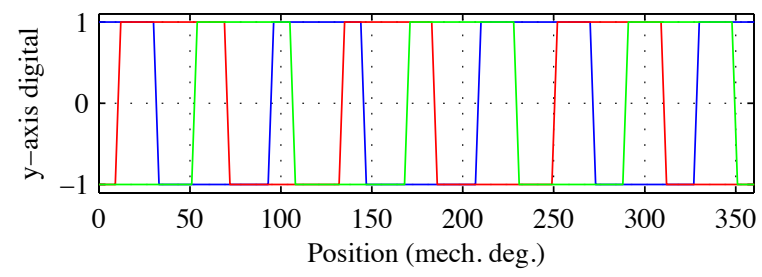

a)

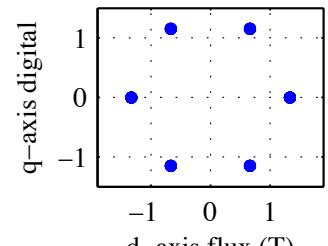

b)

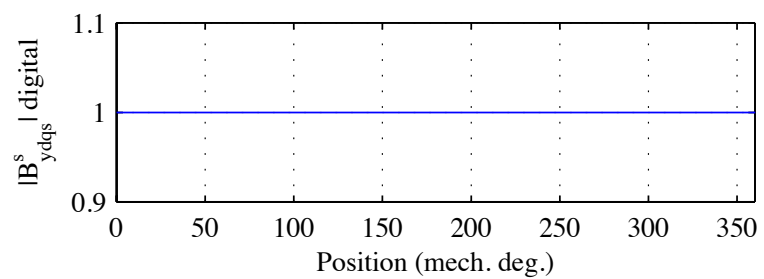

c)

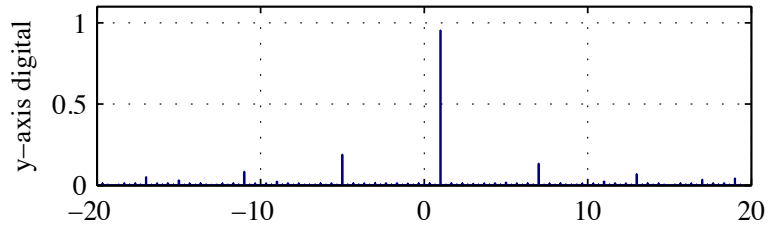

d)

Harmonic order

Fig. 11.- Same results as in Fig. 10 but for the $y$-axis direction.

Fig. 12b shows same results as Fig. 12a for the case of analog hall-effect sensors. It can be observed that the THD both in the $x$ and $y$-axis increases with the demagnetization level. However, demagnetization levels up to $15 \%$, i.e. magnetization state of PM \#5 from $100 \%$ to $85 \%$ (cases \#1$\# 4$ ), can be difficult to distinguish, as the increase of the THD with respect to the healthy case is relatively small. When demagnetization becomes more severe, $(\geq 20 \%$, cases $\# 5-\# 7$ in table III), increase of the THD becomes evident, meaning that the fault can be reliably detected. It is also observed that though both $x$ and $y$-axis show similar trends, measurements in the $y$-axis consistently shows an increased sensitivity.

Fig. 12c shows the mean value of $\left|\bar{B}_{\text {mdqs }}^{s}\right|$ only for the case of analog hall-effect sensors. It is observed that the mean value of $\left|\bar{B}_{\text {mdqs }}^{s}\right|$ decreases as the magnetization state decreases; this was expected since the overall magnetization of the machine decreases. Again the variation of the mean value of $\left|\bar{B}_{m d q s}^{s}\right|$ is observed to be slightly higher when the field is measured along the $y$-axis compared to the $x$-axis, which is consistent with the behavior observe for the THD.

Fig. 12d shows the peak-to-peak value of $\bar{B}_{m d q s}^{s}$ for the case of analog hall-effect sensors. The peak-to-peak value is observed to increase as the magnetization state of PM \#5 decreases. Consistently with the results shown in Fig. 12a and $12 \mathrm{~b}$ demagnetization between 0 and $15 \%$ can be hardly detected.

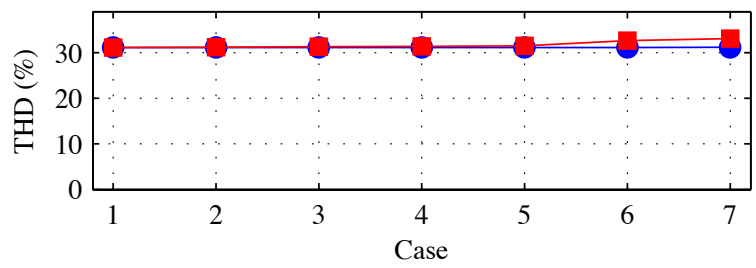

a)

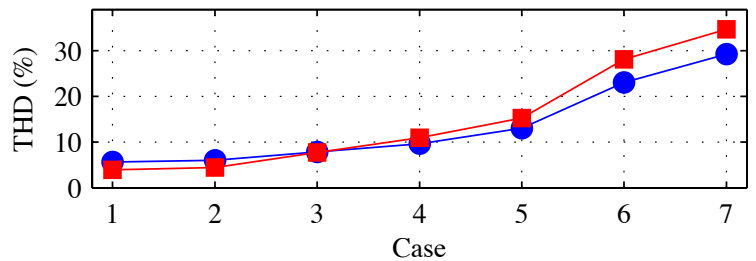

b)

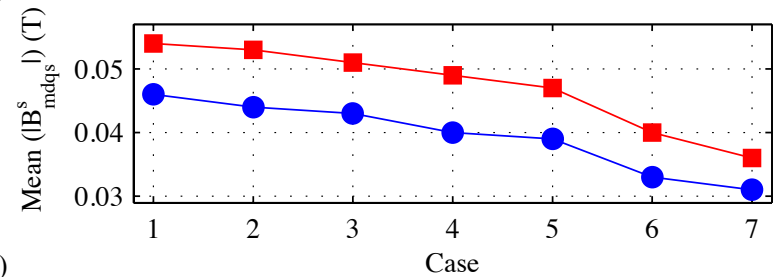

c)

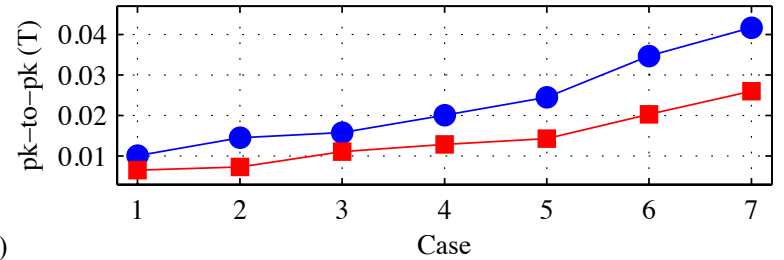

Fig. 12.- Analysis of the results for different cases of demagnetization (see Table III). THD of $\bar{B}_{m d q s}^{s}$ using a) digital hall-effect sensors and, b) analog hall-effect sensors; c) mean value of $\left|\bar{B}_{\text {mdqs }}^{s}\right|$ using analog hall-effect sensors and d) peak-to-peak value of $\vec{B}_{\text {mdqs }}^{s}$ using analog hall-effect sensors. Flux measured in the $x$-axis $(\bullet)$ and $y$-axis $(\square)$.

\section{III.C Machine with asymmetric fault: multiple PMs partially demagnetized}

Fig. 13-Fig. 14 show experimental results when three of the PMs (PMs \#4, \#5 and \#6 in Fig. 1, case \#8 in Table III) are partially demagnetized; Fig. 13 shows the results using analog hall-effect sensors along the $x$-axis direction, Fig. 14 along the $y$-axis direction. It can be observed form Fig. 13a and Fig. 14a that while the peak values of the waveforms are affected by the demagnetization, zero crossings remain almost invariant. Consistently with the experiments shown in Fig. 8 and Fig. 9, measurements in the $y$-axis are significantly more sensitive to demagnetization than the $x$-axis. As expected, the trajectories of $\bar{B}_{x d q s}^{s}$ and $\bar{B}_{y d q s}^{s}$ are not circular anymore (see Fig. 13b and Fig. 14b). Fig. 13c and Fig. 14c show $\left|\bar{B}_{x d q s}^{s}\right|$ and $\left|\bar{B}_{y d q s}^{s}\right|$ while Fig. $13 \mathrm{~d}$ and Fig. $14 \mathrm{~d}$ show the corresponding frequency spectrums. The THD increases by an amount of $\approx 8.76 \%$ for the $y$-axis and $\approx 4.61 \%$ for the $x$-axis with respect to the healthy machine (case \#1).

Experimental results using digital hall-effect sensors are not included since no significant differences exist with respect to case \#7 discussed previously. Table III, case \#8, summarizes the results. 


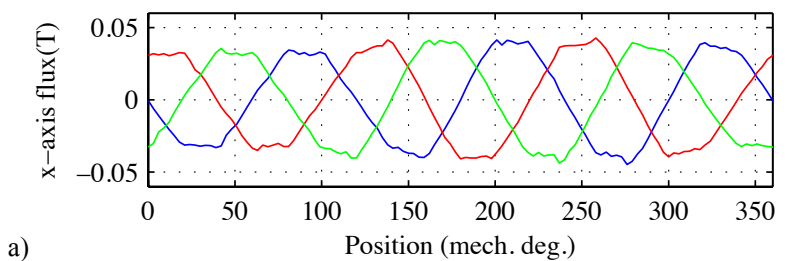

a)

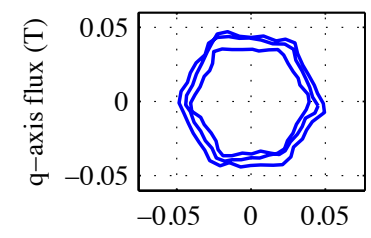

b)
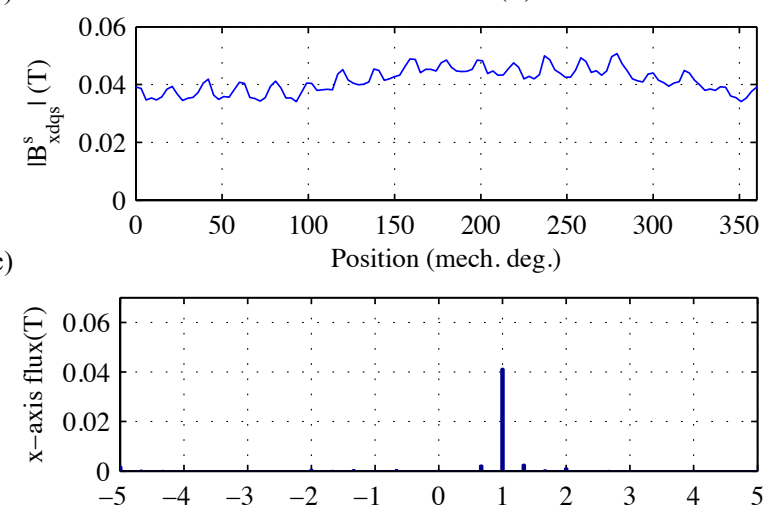

d)

Harmonic order

Fig. 13.- Same results as in Fig. 3 for the case when PMs \#4, \#5 and \#6 (see Fig. 1) magnetization is $0.9 \mathrm{pu}$ (1.15 T, case \#8 in Table III). $\mathrm{x}=0 \mathrm{~mm}, \mathrm{y}=0 \mathrm{~mm}, \mathrm{z}=5 \mathrm{~mm} . \omega_{r}=1 \mathrm{pu}$ and $i_{d q}=0 \mathrm{pu}$.

Two cases using two magnets partially demagnetized (PM \#5 and \#6 in Fig. 1), cases \#9 and \#10 in Table III, have also been considered.

It can be observed from Table III that, consistently with the experimental results shown in Section III.B (cases \#2-\#5), differences in the THD, $x$ and $y$-axis, are almost negligible when the demagnetization is $<15 \%$, differences among one, two or three partially demagnetized PMs cases being hardly detectable. As expected, the mean magnitudes of $\left|\bar{B}_{x d q s}^{s}\right|$ and $\left|\bar{B}_{y d q s}^{s}\right|$ decrease as the number of demagnetized PMs increases (for the same demagnetization degree). THD, $x$ and $y$-axis, is seen to slightly depend on the number of demagnetized PMs, while the peak-to-peak value is not affected, which was an expected result.

III.D Machine with all PMs partially demagnetized (symmetric fault)

Experiment with all PMs partially demagnetized was carried out $(80 \%$ of the rated magnetization state, case \#11 in Table III). It is observed from Table III that the differences in the THD, $x / y$-axis, digital/analog hall-effect sensors, between the healthy machine (case \#1 in Table III) and this case are negligible. It is also observed that the mean magnitudes of $\left|\bar{B}_{x d q s}^{s}\right|$ and $\left|\bar{B}_{y d q s}^{s}\right|$ decrease by $\approx 20 \%$ respect the healthy machine, i.e. symmetric demagnetization fault can be reliable detected by the mean magnitude of $\left|\bar{B}_{x d q s}^{s}\right|$ and $\left|\bar{B}_{y d q s}^{s}\right|$. On the contrary, it is observed from Table III that symmetric demagnetization cannot be reliable detected using digital halleffect sensors. This was an expected result as symmetric
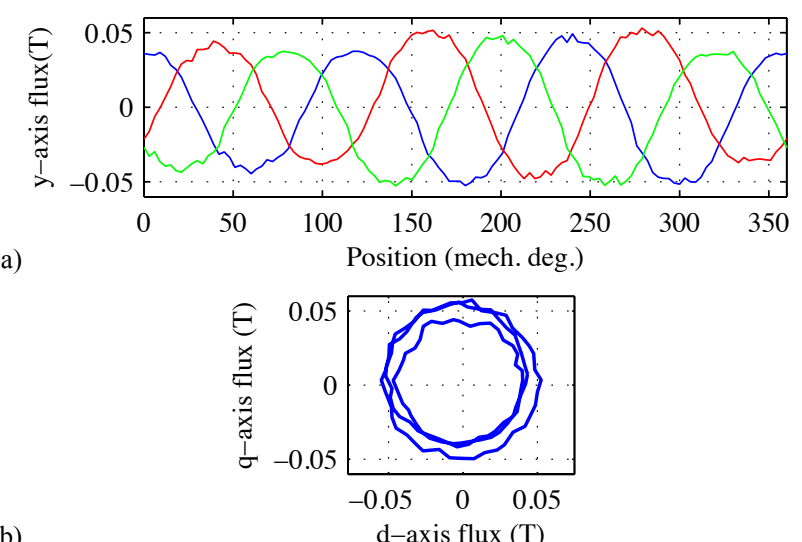

b)
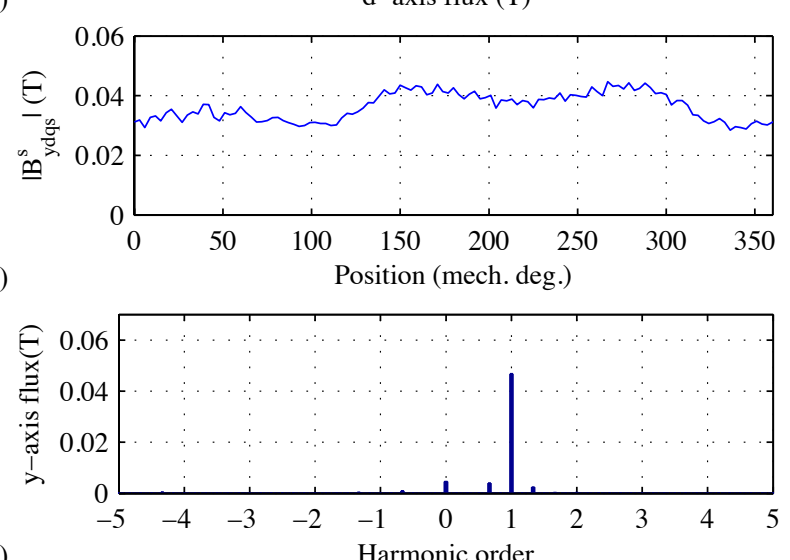

Fig. 14.- Same results as in Fig. 13 but for the $y$-axis direction.

demagnetization modifies the magnitude of the flux vector, but magnitude information is lost during digitalization.

Results of all the experiments that have been performed are summarized in the right columns of Table III. Two major conclusions are reached: 1) $y$-axis is the preferred direction to measure the field for demagnetization detection purposes; 2) demagnetization detection is significantly more challenging using digital sensors compared to the case of analog sensors. It is interesting to note in this regard that while retrofit of existing drives (machine and control) to replace digital halleffect sensors by analog devices is not considered viable in most of applications due to economic issues, this would be perfectly possible in new designs. Indeed analog sensors are not necessarily more expensive than digital sensors, also modern digital signal processors offer variants with increased analog inputs at very competitive costs.

\section{Implementation issues}

There are a number of issues, which could affect to the performance of the proposed method and must therefore be considered. These include, among others: 1) magnets' temperature, 2) offsets in the sensors, 3) variations in the sensors' gains; 4) assembling tolerances and 5) stator current effects. All these issues are analyzed following. The following discussion will be limited to the case of analog sensors, as this option provides significantly better results than digital sensors.

\section{IV.A. Effect of magnet temperature}


PM temperature makes the PM remanent flux, $B_{r}$, to change, according the PM thermal remanent flux coefficient, which is defined as the PM remanent flux rate of variation with temperature, $\alpha_{B}$ [2] and [6]. Typically, an increase of PM temperature results in a reduction of $B_{r}$, i.e. the magnet becomes demagnetized. If the PM's temperature becomes higher than its maximum working temperature, the PM will become irreversible demagnetized. Otherwise demagnetization will be reversible, i.e. the PM recovers its original properties when the temperature decreases.

If the temperature of the rotor magnets is uniform, i.e. no significant difference among poles, an increase in the PMs' temperature will decrease the field, but without any further effect as additional harmonics or THD increase of the field complex vector. This corresponds to case \#11 in Table III.

If the temperature of the rotor magnets is not uniform, i.e. difference among poles exists (e.g. due to rotor lamination grain orientation [33]), the same measurable effect as nonuniform demagnetization will be induced.

It is concluded from the previous discussion that temperature variation will induce the same effects as PM demagnetization. This uncertainty can be avoided if the magnet temperature can be measured or estimated [6].

\section{IV.B. Effects due to offsets in the sensors}

Offsets in the sensors measurements, mainly due to imperfection in the electronics, induce a DC component whose magnitude and phase depend on the offsets [34]. It is observed from Fig. 8 and 14, that demagnetization also induces a DC component. To prevent interference of the sensor's offsets with the method, a pre-commissioning stage to calibrate the sensors can be performed.

It must be noted however that this effect will be only relevant if demagnetization detection is based on the absolute value, e.g. of THD or peak-to-peak values. On the contrary, if demagnetization detection is based on the detection of incremental variation with respect to the healthy case, offsets in the sensors are not expected to affect.

\section{IV.C. Effects due to unbalances in sensors gains}

Unbalances in sensors' gains will affect to the fundamental component of $\bar{B}_{m d q s}^{s}$, and also induce a negative sequence component [34]. Demagnetization faults also induce negative sequence component in $\bar{B}_{m d q s}^{s}$ spectrum. However, as discussed in the previous subsection, this is not expected to affect to the performance of the method assumed that demagnetization detection is based on incremental variations form the healthy case.

\section{IV.D. Effects due to assembling tolerances}

Assembling tolerances in the sensors assembling include displacements in axial, radial and angular directions.

Axial and radial displacements induce a variation of the magnetic flux density measured by the sensor, i.e. it can be modeled as a variation of the sensor's gain [34], which has been previously analyzed in section $I V-C$.

Angular displacements induce four additional components to $\bar{B}_{\text {mdqs }}^{s}$ spectrum [34]: two positive sequence components and two negative sequence components. Conclusions obtained for the case of unbalances in the sensors' gains apply in this case.

\section{IV.E. Effects due to stator current}

\section{a) Stator d-axis current}

Flux due to negative $d$-axis current partially counteracts the PM flux (flux-weakening current), resulting in a reduction in the magnetic flux density measured by the hall-effect sensors [34]. On the other hand, negative $d$-axis current does not induce significant variations in $\bar{B}_{m d q s}^{s}$ harmonic content [34]. It can be concluded from the previous discussion that negative $d$-axis current produce the same effect as a uniform temperature increase or uniform demagnetization in the PMs, see section $I V-A$, meaning that distinguishing among these effects might not be feasible. However, it is possible to measure the influence of the $d$-axis current during a commissioning process and further compensate for it during normal operation of the drive.

\section{b) Stator q-axis current}

Flux induced by $q$-axis current results in slight variations of $\left|\bar{B}_{x d q s}^{s}\right|$ mean value and of $\bar{B}_{\text {mdqs }}^{s}$ harmonic content [34]. Since uniform PM temperature variation and negative $d$-axis current also induce variations of $\left|\bar{B}_{x d q s}^{s}\right|$ mean value, the conclusions reached in those cases (section $I V-A$ and $I V-E$ ) apply. Asymmetric PM demagnetization faults also increase $\bar{B}_{m d q s}^{s}$ harmonic content; therefore $q$-axis current injection could limit the reliability of the proposed demagnetization detection method. However, the THD increase due asymmetric PM demagnetization faults is expected to be more significant compared to $I_{q}$ current injection (e.g. $\approx 3.5 \%$

\begin{tabular}{|c|c|c|c|c|c|c|c|c|c|c|c|c|c|c|}
\hline & \multicolumn{14}{|c|}{ Table III. Experimental results } \\
\hline \multirow{3}{*}{ Case } & \multirow{2}{*}{\multicolumn{6}{|c|}{ PM magnetization state }} & \multicolumn{4}{|c|}{$x$-axis measurement } & \multicolumn{4}{|c|}{$y$-axis measurement } \\
\hline & & & & & & & \multicolumn{2}{|c|}{ THD (\%) } & \multicolumn{2}{|c|}{$(\mathrm{T})$} & \multicolumn{2}{|c|}{ THD (\%) } & \multicolumn{2}{|c|}{$(\mathrm{T})$} \\
\hline & $\# 1$ & $\# 2$ & $\# 3$ & $\# 4$ & $\# 5$ & $\# 6$ & Digital & Analog & Mean & pk-to-pk & Digital & Analog & Mean & pk-to-pk \\
\hline$\# 1$ & 100 & 100 & 100 & 100 & 100 & 100 & 31.6 & 5.63 & 0.046 & 0.0101 & 31.6 & 3.98 & 0.054 & 0.0065 \\
\hline$\# 2$ & 100 & 100 & 100 & 100 & 95 & 100 & 31.6 & 6.02 & 0.044 & 0.0145 & 31.18 & 4.47 & 0.053 & 0.0073 \\
\hline$\# 3$ & 100 & 100 & 100 & 100 & 90 & 100 & 31.6 & 7.89 & 0.043 & 0.0158 & 31.31 & 7.77 & 0.051 & 0.0111 \\
\hline$\# 4$ & 100 & 100 & 100 & 100 & 85 & 100 & 31.6 & 9.63 & 0.04 & 0.0201 & 31.38 & 10.98 & 0.049 & 0.0129 \\
\hline$\# 5$ & 100 & 100 & 100 & 100 & 80 & 100 & 31.6 & 13.04 & 0.039 & 0.0245 & 31.48 & 15.25 & 0.047 & 0.0143 \\
\hline$\# 6$ & 100 & 100 & 100 & 100 & 60 & 100 & 31.6 & 23.07 & 0.033 & 0.0347 & 32.62 & 28.08 & 0.04 & 0.0203 \\
\hline$\# 7$ & 100 & 100 & 100 & 100 & 50 & 100 & 31.7 & 29.22 & 0.031 & 0.0417 & 33.11 & 34.7 & 0.036 & 0.026 \\
\hline$\# 8$ & 100 & 100 & 100 & 90 & 90 & 90 & 31.6 & 10.76 & 0.034 & 0.016 & 31.58 & 13.8 & 0.043 & 0.0156 \\
\hline$\# 9$ & 100 & 100 & 100 & 100 & 90 & 90 & 31.6 & 8.58 & 0.041 & 0.0161 & 31.21 & 9.13 & 0.05 & 0.0125 \\
\hline$\# 10$ & 100 & 100 & 100 & 100 & 90 & 80 & 31.6 & 15.96 & 0.037 & 0.0249 & 31.56 & 17.01 & 0.045 & 0.0187 \\
\hline$\# 11$ & 80 & 80 & 80 & 80 & 80 & 80 & 31.6 & 5.37 & 0.035 & 0.0094 & 31.6 & 3.89 & 0.042 & 0.0056 \\
\hline
\end{tabular}


increase for $I_{q}=1 \mathrm{pu}$ and for the test machine shown in Fig. 1 [34]). Therefore $I_{q}$ current injection effect is expected to be of reduced importance in practice.

\section{Conclusions}

The use of low cost hall-effect sensors for PMSMs demagnetization detection is proposed in this paper. Preliminary experimental results show that while both analog and digital hall-effect sensors can potentially be used for this purpose, analog type are clearly advantageous. This was an expected result as conversion of a continuous signal into a digital signal, which can only take two values necessarily, implies an information loss. It has also been determined that alignment of the field sensor with the radial direction improves the sensitivity of the method. The THD and the peak-to-peak value of the magnetic flux density complex vector have been shown to be reliable metrics, the second one being much simpler to obtain in practice. Implementation issues which could affect to the performance of the proposed method have also been discussed, including magnets' temperature, offsets in the sensors, variations in the sensors' gains, assembling tolerances and stator current effects.

It is finally noted that the proposed method has been discussed for machines equipping three hall-effect sensors, which is a common choice in commercial PMSMs. However it can also be implemented using only two hall-effect sensors shifted by 90 electrical degrees.

\section{References}

[1] D. W. Novotny and T. A. Lipo, "Vector Control and Dynamics of AC Drives," Oxford Science Publications, 1996.

[2] J. F. Gieras and M. Wing, "Permanent magnet motor technology: design and application". Second edition 2002.

[3] S. Ruoho, J. Kolehmainen, J. Ikaheimo and A. Arkkio,"Interdependence of Demagnetization, Loading, and Temperature Rise in a PermanentMagnet Synchronous Motor," IEEE Trans. Mag., 46(3): 949-953, Mar. 2010.

[4] J. Hong, D. Hyun, and S. B. Lee, "Automated monitoring of magnet synchronous motors at standstill," IEEE Trans. Ind. Appl., 46 (4): 1397 $1405,2010$.

[5] IEC 62. 2-2004. "IEEE guide for diagnostic field testing of electric power apparatus-electrical machinery," 2004.

[6] D. Reigosa, D. Fernandez, H. Yoshida, T. Kato and F. Briz, "PermanentMagnet Temperature Estimation in PMSMs Using Pulsating HighFrequency Current Injection,” IEEE Trans. on Ind. Appl., 51(4): 31593168, July-Aug 2015.

[7] X. Xiao, C. Chen and M. Zhang, "Dynamic Permanent Magnet Flux Estimation of Permanent Magnet Synchronous Machines", IEEE Trans. on Appl. Sup., 20(3): 1085-1088, June. 2010.

[8] T. J. Vyncke, R. K. Boel and J. A. A. Melkebeek, "A Comparison of Stator Flux Linkage Estimators for a Direct Torque Controlled PMSM Drive", IEEE IECON 20(3): 971-978, Nov. 2009.

[9] K. Liu and Z. Q. Zhu "Online Estimation of the Rotor Flux Linkage and Voltage-Source Inverter Nonlinearity in Permanent Magnet Synchronous Machine Drives," IEEE Trans. on Power Elect., 29(1): 418-427, Jan. 2014.

[10] K. Liu, Q. Zhang, J. Chen, Z. Q. Zhu, and J. Zhang, "Online multiparameter estimation of nonsalient-pole PM synchronous machines with temperature variation," IEEE Trans. Ind. Electron., 58(5): 17761788, May 2011.

[11] D. Reigosa, D. Fernandez, J. M. Guerrero, Z.Q. Zhu and F. Briz, "PMSM Magnetization State Estimation Based on Stator-reflected PM Resistance Using High Frequency Signal Injection”, IEEE Trans. on Ind. Appl., 51(5): 3800-3810, Sept.-Oct. 2015.

[12] D. Fernandez, D. Reigosa, Z. Q. Zhu and F. Briz, "Permanent-Magnet Magnetization State Estimation Using High-Frequency Signal
Injection”, IEEE Trans. on Ind. Appl., 52(4): 2930-2949, July-Aug. 2016.

[13] J.-R. R. Ruiz, A. G. Espinosa, L. Romeral, and J. Cusidó, "Demagnetization diagnosis in permanent magnet synchronous motors under nonstationary speed conditions," Electr. Power Syst. Res., 80(10): 12771285, Oct. 2010.

[14] A. G. Espinosa, J. A. Rosero, J. Cusido, L. Romeral, and J. A. Ortega, "Fault detection by means of Hilbert-Huang transform of the stator current in a PMSM with demagnetization," IEEE Trans. Energy Convers., 25(2): 312-318, Jun. 2010.

[15] C. Wang, M. Delgado, L. Romeral, Z. Chen, F. Blaabjerg and X. Liu," Detection of Partial Demagnetization Fault in PMSMs Operating Under Non stationary Conditions," IEEE Trans. Magn., 52(7), July 2016.

[16] W. le Roux, R. G. Harley, and T. G. Habetler, "Detecting rotor faults in low power permanent magnet synchronous machines," IEEE Trans. Power Electron., 22(1): 322-328, Jan. 2007.

[17] S. Rajagopalan, W. le Roux, T. G. Habetler, and R. G. Harley, "Dynamic eccentricity and demagnetized rotor magnet detection in trapezoidal flux (brushless DC) motors operating under different load conditions,” IEEE Trans. Power Electron., 22(5): 2061-2069, Sep. 2007.

[18] C. Urresty, J.-R. R. Ruiz, M. Delgado, and L. Romeral, "Detection of demagnetization faults in surface-mounted permanent magnet synchronous motors by means of the zero-sequence voltage component," IEEE Trans. Energy Convers., 27(1): 42-51, Mar. 2012.

[19] J. C. Urresty, J.-R. R. Ruiz, and L. Romeral, "A back-EMF based method to detect magnet failures in PMSMs," IEEE Trans. Mag., 49(1): 591-598, Jan. 2013

[20] Z. Yang, X. Shi, and M. Krishnamurthy, "Vibration monitoring of PM synchronous machine with partial demagnetization and inter-turn short circuit faults," in Proc. IEEE ITEC, pp. 1-6, June 2014.

[21] D. Torregrossa, A. Khoobroo and B Fahimi, "Prediction of Acoustic Noise and Torque Pulsation in PM Synchronous Machines With Static Eccentricity and Partial Demagnetization Using Field Reconstruction Method", IEEE Trans. on Ind. Elect., 59(2): 934-944, Feb. 2012.

[22] J. C. Urresty, R. Atashkhooei, J.-R. R. Ruiz, L. Romeral, and S. Royo, "Shaft trajectory analysis in a partially demagnetized permanent-magnet synchronous motor," IEEE Trans. Ind. Electron., 60(8): 3454-3461, Aug. 2013.

[23] J. Hong, D. Hyun, S.B. Lee, J.Y. Yoo and K.W. Lee, "Automated Monitoring of Magnet Quality for Permanent-Magnet Synchronous Motors at Standstill," IEEE Trans. Ind. Appl., 46(4): 1397-1405, JulyAug. 2010.

[24] Maxonmotorusa.com, "maxon sensor - Key information," Mar. 23, 2017. [Online]. Available: http://www.maxonmotorusa.com/

[25] abbmotion.com, "Inteligent Servo Drives and Inteligent Motors," MotiFelex e180 motion control, Mar. 23, 2017. [Online]. Available: http://www.abbmotion.com/products/servodrives/overview.asp

[26] F. G. Capponi, G. D. Donato, L. D. Ferraro, O. Honorati, M. C. Harke, and R. D. Lorenz, "AC brushless drive with low-resolution hall-effect sensors for surface-mounted PM machines," IEEE Trans. Ind. Appl., vol. 42, no. 2, pp. 526-535, March/April 2006.

[27] S. Morimoto, M. Sanada, and Y. Takeda, "High performance currentsensorless drive for PMSM and SynRM with only low resolution position sensor," IEEE Trans. Ind. Appl., 39(3): 792-801, May/Jun. 2003.

[28] faulhaber.com, "Brushless De servomotors," Series 3268...BX4, Mar. 23, 2017. [Online]. Available: https://fmcc.faulhaber.com

[29] micromo.com, "Micro Motion systems, Series 2036", October 11, 2017. [Online]. Available: http://www.micromo.com

[30] X. Song, J. Fang, and B. Han, "High-Precision Rotor Position Detection for High-Speed Surface PMSM Drive Based on Linear Hall-Effect Sensors," IEEE Trans. Power Electron., 31(7): 4720 -4731, July 2016.

[31] Y. Y. Lee, R.-H. Wu, and S.T. Xu, "Applications of Linear Hall-Effect Sensors on Angular Measurement," IEEE International Conference on Control Applications (CCA), pp. 479 -482, Sept. 2011

[32] L. Xiao, Y. Yunyue, Z. Zhuo, "Study of the Linear Hall-Effect Sensors Mounting Position for PMLSM," IEEE Conference on Industrial Electronics and Applications, pp. 1175 - 1178, May 2017.

[33] D. Reigosa, D. Fernandez, T. Tanimoto, T. Kato and F. Briz, "Sensitivity Analysis of High Frequency Signal Injection Based Temperature Estimation Methods to Machine Assembling Tolerances," IEEE Trans. on Ind. Appl., 52(6): 4798-4805, Nov-Dec 2016.

[34] D. Reigosa, D. Fernandez, C. Gonzalez, S. B. Lee and F. Briz, "Permanent Magnet Synchronous Machine Drive Control Using Analog Hall-Effect Sensors", IEEE Trans. Ind. Appl., 54(3): early access article, May-June. 2018. 
[35] D. Reigosa, D. Fernandez, Yonghyun Park, A. B. Diez, S. B. Lee and F. Briz, "Detection of Demagnetization in Permanent Magnet Synchronous Machines Using Hall-Effect Sensors", IEEE-ECCE'17, pp. 4686-4693, Oct 2017. 